\title{
Defining Global Supervenience
}

\begin{abstract}
What does it mean that certain properties globally supervene on others? The paper criticises the now standard way of spelling out the notion in terms of 1-1 correlations between world-domains and proposes a modification that escapes the difficulties. The new definition can secure the additional benefit of resisting an argument to the effect that global supervenience is theoretically dispensable.
\end{abstract}

The rough and ready slogan of supervenience is that $A$-properties supervene on $B$-properties just in case there cannot be a difference in $A$-properties without a difference in $B$-properties. As Jaegwon Kim (1984) pointed out the slogan may be understood in at least three ways. Correspondingly, he distinguished three kinds of supervenience, a weak and a strong form of individual supervenience and global supervenience.

Individual supervenience deals in property distributions across individuals, global supervenience in property distributions across whole possible worlds. Roughly, $A$-properties globally supervene on $B$-properties just in case whenever worlds agree in their distribution of $B$-properties, they also agree in their distribution of $A$-properties. But when do worlds agree in their distribution of some kind of properties? Philosophers have favoured different analyses of this ingredient notion of global supervenience. In section 1 I give a brief sketch of these elucidations and the resulting notions of global supervenience. In section $2 \mathrm{I}$ argue that the analysis favoured in the current literature is inadequate as a foundation for satisfactory global supervenience notions. Section 3 proposes a better understanding. Section 4 shows how the resulting notions of global supervenience can secure the additional benefit of resisting an argument to the effect that global supervenience is theoretically dispensable.

\section{Global Supervenience}

Sometimes the world-wide distribution of one family of properties $B$ fixes the world-wide distribution of another family of properties $A$, even when 
the $B$-properties of a possible individual do not fix its $A$-properties. This is where global supervenience comes into its own. Consider, for instance, the sets of properties MAss and MostMass. A property is an element of MASS just in case it is the property of having a mass of $x \mathrm{~kg}$, for some number $x$. MostMass is the unit set of the property of having at least as much mass as anything else. Now, intuitively, worlds that agree in the distribution of MAss-properties agree in their distribution of the MostMAss-property. If both at $w_{1}$ and at $w_{2}$ Fred has the property of having a mass of 80 $\mathrm{kg}$ and nothing has the property of having a mass of $80+y \mathrm{~kg}$ for some positive number $y$, then Fred has the property of weighing at least as much as anything else at both worlds. This generalises: MostMass globally supervenes on MAss-properties. However, MostMass does not (strongly) individually supervene on MASS-properties: consider a further world $w_{3}$ which is like $w_{2}$ apart from containing an extra thing, Frank, that has a mass of $100 \mathrm{~kg}$. At both $w_{1}$ and $w_{3}$, Fred has exactly the same MAss-properties. But at $w_{3}$, Fred lacks the MostMAss-property of having at least as much mass as anything else - a property he has at $w_{1}$-because of Frank: Fred's MAss-properties do not fix his MostMAss-property. ${ }^{1}$ A (philosophical) real life example in which global but not individual supervenience may be present is the mental and the physical, given that mental content is partly determined externally.

But what exactly does it mean that worlds agree in their distribution of $A$-properties? In the early days of the debate, Kim (1984: 167f.) offered the following: ${ }^{2}$

\section{World-Agreement (Kim)}

$w$ and $w^{\prime}$ agree in their distribution of $A$-properties $\leftrightarrow_{\mathrm{df}}$. $\forall P \in A \forall x\left(\right.$ at $w, x$ has $P \leftrightarrow$ at $w^{\prime}, x$ has $P$ )

In words: $w$ and $w^{\prime}$ agree in their distribution of $A$-properties just in case whatever has an $A$-property in one of the worlds has it in the other. We can plug this into our informal understanding of global supervenience. This results in the claim that $A$-properties globally supervene on $B$-properties just in case whenever there are worlds at one of which a possible individual has an $A$-property it lacks at the other, some possible individual also has different $B$-properties at these worlds.

\footnotetext{
${ }^{1}$ For a precise characterisation of the notion of strong individual supervenience see section 4 below.

${ }^{2}$ I follow the bulk of the supervenience debate in framing the issues in terms of possible worlds. Correlatively, the objectual quantifiers are possibilist, i.e. they range over all possible individuals.
} 
Kim's original understanding has, however, been replaced by an (at least in some respects) more lenient understanding of what it means for worlds to agree in their distribution of $A$-properties. ${ }^{3}$ The complaint about the original proposal is that it only applies to worlds at which numerically the same individuals have the properties in question, raising doubts as to its flexibility as well as its applicability on accounts of possibilia on which individuals are world-bound (see e.g. McLaughlin 1995: 32). ${ }^{4}$ Instead, the thought is, we should only require that there is a bijection - a one-one correspondencefrom the inhabitants of one world (the so-called world-domain) onto the inhabitants of the other that preserves $A$-properties (with respect to these two worlds). If both worlds have the very same individuals in their worlddomains, the identity-function may be such a bijection, but there are other candidates, too. The proposal is thus:

\section{World-Agreement}

$w$ and $w^{\prime}$ agree in their distribution of $A$-properties $\leftrightarrow_{\text {df. }} \exists f$

(i) $\quad f: \mathfrak{d}(w) \mapsto \mathfrak{d}\left(w^{\prime}\right)$;

(ii) $f$ is bijective;

(iii) $\forall P \in A \forall x \in \mathfrak{d}(w)$ (at $w, x$ has $P \leftrightarrow$ at $w^{\prime}, f(x)$ has $P$ )

If some function satisfies clause (i) and (ii) for $w$ and $w^{\prime}$, it is a bijection between their world-domains. If, moreover, this function satisfies clause (iii) with respect to $w$ and $w^{\prime}$, it is $A$-preserving (with respect to $w$ and $w^{\prime}$ ). For brevity I will thus talk about $A$-preserving bijections.

Plugging this new understanding of what it means for worlds to agree in their distribution of some kind of properties into our informal understanding of global supervenience results in what is known as weak global supervenience:

\section{Weak Global Supervenience}

$A$-properties weak-globally supervene on $B$-properties $\leftrightarrow_{\mathrm{df}}$. For any worlds $w$ and $w^{\prime}$ : If there is a $B$-preserving bijection from $\mathfrak{d}(w)$ to $\mathfrak{d}\left(w^{\prime}\right)$, then there is an $A$-preserving bijection from $\mathfrak{d}(w)$ to $\mathfrak{d}\left(w^{\prime}\right)$.

Weak Global Supervenience leaves it open whether there is a connection between the $A$ - and $B$-preserving bijections: it allows but does not require them to be the same function. By requiring there to be some such

\footnotetext{
${ }^{3} \operatorname{Kim}$ (1988: §3) gestures in that direction. Paull and Sider (1992); McLaughlin (1995); Stalnaker (1996); McLaughlin (1997); Sider (1999); Shagrir (2002); Bennett (2004) pursue the idea more rigorously.

${ }^{4}$ The locus classicus pro world-boundness is Lewis (1986). Whether the complaints are justified need not be decided here.
} 
connection more demanding notions of supervenience may be defined, the most straightforward among which figuring under the names intermediate and strong global supervenience. ${ }^{5}$ In the recent literature on supervenience, these notions are univocally taken to be the prime candidates for what we may reasonably have in mind when we speak unguardedly of global supervenience. However, in the next section I argue that they are inadequate due to a gratuitous restriction of the purview of their ingredient notion of worldagreement. I discuss only the case of Weak Global Supervenience explicitly. The other notions of supervenience will be guilty at least by association. ${ }^{6}$

\section{Scope Limitations}

In this section I argue that two worlds can satisfy the definiens of WorldAgreement for $A$-properties while disagreeing in their distribution of $A$-properties, since the definiens ignores what properties the non-existents of a world have or lack at that world. Since this is an arbitrary restriction of the purview of a notion of world-agreement and the corresponding notions of global supervenience, it should be lifted.

But hold on, 'the properties the non-existents at a world have or lack'? Surely there are no such things! Surely, that is, all properties are existenceentailing in the following sense: ${ }^{7}$

\section{Existence Entailment}

A property $P$ is existence-entailing $\leftrightarrow_{\mathrm{df}}$.

$\forall x \forall w($ at $w, x$ has $P \rightarrow$ at $w, x$ exists $)$

In what follows I argue that (i) the claim that some modal properties are not existence-entailing follows from plausible principles and that (ii) those properties that are not existence-entailing spell trouble for World-Agreement.

The first assumption the argument relies on is the following:

A1 There are worlds $w$ and $w^{\prime}$ and a possible object $x$ such that

(i) $\quad w^{\prime}$ has access to $w$;

\footnotetext{
${ }^{5}$ Cp. e.g. Bennett (2004: 503).

${ }^{6}$ The argument from modal properties below has a less striking analogue for intermediate but none for strong global supervenience. However, the three notions of global supervenience, being all built on the same notion of world-agreement, come in a pleasingly uniform package. All else being equal, if one receives modification, so should the others.

${ }^{7}$ The nomenclature is borrowed from Plantinga (1983: 15). The claim that all properties are existence-entailing is sometimes called serious actualism. Cp. Plantinga (1983: $6)$.
} 
(ii) at $w, x$ is $F$; with ' $F$ ' some general term you like, perhaps 'philosopher'

(iii) at $w^{\prime}, \neg(x$ exists $)$.

If you think that some objects that are actually philosophers might have failed to exist and that accessibility is symmetric, your beliefs entail the truth of (A1). For, take any philosopher who might have failed to exist, perhaps you. You are a philosopher at the actual world @, you don't exist at some world accessible from @, and, since accessibility is symmetric, @ is accessible from that world.

Consequently, the only possible reasons I can see to deny (A1) are (i) by appeal to necessitarianism, the view that every possible object exists at every possible world, or (ii) by the adoption of a very weak modal logic whose soundness does not even rely on accessibility's being symmetric. Neither of these option seems particularly attractive. ${ }^{8}$ In any case, it is a sound methodological maxim not to make the adequacy of one's definitions to depend on such substantial (and highly controversial) moves.

To get from (A1) to the conclusion that some modal properties are not existence-entailing, we need three transition principles: one to get from possible worlds to modal sentence operator talk, one to get from modal sentence operator talk to modal predications, and one to get from predications to property ascriptions. They read as follows:

A2 $\forall w \forall p\left(\right.$ at $w, \diamond p \leftrightarrow \exists w^{\prime}\left(w\right.$ has access to $w^{\prime} \wedge$ at $\left.\left.w^{\prime}, p\right)\right)$

A3 $\forall w \forall x \forall F($ at $w, \diamond(x$ is $F) \leftrightarrow$ at $w, x$ is $\langle\lambda y \diamond(y$ is $F)\rangle)$

A4 $\forall F$ (If there is such a thing as the property of being $F$, then $\forall w \forall x$ (at $w, x$ is $\langle\lambda y y$ is $F\rangle \leftrightarrow$ at $w, x$ has the property of being $F$ ))

(A2) is a familiar principle that is partly constitutive of our understanding of possible worlds. (A3) makes an assumption explicit that is typically taken for granted in modal logics whose languages are too coarse to make the distinction between sentence and predicate modifier uses of modal operators. ${ }^{9}$ In systems that have the resources to represent the difference, (A3)

\footnotetext{
${ }^{8}$ For a defense of necessitarianism, see, e.g., Linsky and Zalta (1994) and Williamson (2002), however.

${ }^{9}$ ' $\lambda y$ ' in the pertinent use is a general-term-forming operator on open sentences. The syntactic difference between ' $\nabla(a$ is $F)$ ' and ' $a$ is $\langle\lambda y \diamond(y$ is $F)\rangle$ ' corresponds to the difference between 'It is possible that Socrates is a carpenter' on the one hand and 'Socrates is possibly a carpenter' or, more explicitly, 'Socrates is such that he is possibly a carpenter' on the other.
} 
comes out as a theorem. ${ }^{10}$ (A4), finally, articulates a connection between simple predications and property ascriptions that is built into the most straightforward abundant conception of properties. ${ }^{11}$ Since the supervenience debate needs just such a conception, I take (A4) to be justified as well. All three principles may, perhaps, be reasonably doubted. But the adequacy of supervenience notions should not be made to depend on such prima facie unattractive moves. I will first present the argument in detail. Then, in the next section, I propose improvements and show that even a disbeliever in non-existence-entailing properties should prefer the newly introduced notions.

Let ' $F$ ' be any general term for which (A1) is true - plausibly: '(a) philosopher', '(a) person', but not 'prime'. It is now easy to show that the property of being possibly $F$ is not existence-entailing. For, let $w$ and $w^{\prime}$ be any possible worlds and $a$ any possible individual that make the relevant instance of (A1) true. By (i) and (ii) of (A1):

$1 \quad w^{\prime}$ has access to $w \wedge$ at $w, a$ is $F$.

By (A2):

$2 \quad$ at $w^{\prime}, \diamond(a$ is $F)$.

By (A3):

3 at $w^{\prime}, a$ is $\langle\lambda y \diamond(y$ is $F)\rangle$.

By (A4):

4 at $w^{\prime}, a$ has the property of being possibly $F$.

By (iii) of (A1)

$5 \quad$ at $w^{\prime}, \neg(a$ exists $) \wedge$ at $w^{\prime}, a$ has the property of being possibly $F$.

Thus, the property of being possibly $F$ is not existence-entailing.

An argument relying on straightforward variants of (A1) to (A4) works for properties of the being-necessarily- $F$ variety. Thus, modal properties whose demodalisations can be exemplified by contingently existing objects are not existence-entailing.

Now, worlds $w$ and $w^{\prime}$ may differ in their distribution of properties that are not existence-entailing even though they do not disagree in their distribution of these properties over their existents. As a toy example take the

\footnotetext{
${ }^{10}$ See, e.g., Fitting and Mendelsohn (1998: ch. 10).

${ }^{11}$ The antecedent is meant to exclude trouble makers like the Russell property. For a discussion of Plantingan worries about (A4) that I take to be conclusive see Fine (1985: 197ff.).
} 
property signified by the predicate '- exists $\vee$ Socrates exists'. Call it $P$. For any possible world $w$ and possible object $x$ that exists at $w$ : at $w, x$ has $P$ by virtue of existing at $w$. Thus, no two worlds with the same number of existents disagree on the distribution of $P$ over their existents. But worlds may differ with respect to their overall distribution of $P$ by differing with respect to $P$ 's distribution over their non-existents. In particular, let $w$ be any world at which Socrates exists and $w^{\prime}$ any world at which Socrates does not exist. Then, a possible object that does not exist at $w$ has $P$ at $w$ by virtue of Socrates' existing at $w$, but no possible object that does not exist at $w^{\prime}$ has $P$ at $w^{\prime}$, since neither it nor Socrates exists at $w^{\prime}$. Thus any pair of worlds with the same number of existents at only one of which Socrates exists shows that World-Agreement is inadequate.

Correspondingly, the notions of global supervenience defined in terms of World-Agreement are inadequate as well. Intuitively, in our toy example, $\{P\}$ globally supervenes on $\{$ the property of existing, the property of being such that Socrates exists $\}$ but not on an arbitrary set of properties, e.g. not on MAss. But since any two worlds satisfy the definiens of WorldAgreement for $\{P\},\{P\}$ weak-globally supervenes on any set of properties. If there are properties that are not existence-entailing, weak-global supervenience is inadequate because it simply ignores some aspects of the property distributions at worlds. ${ }^{12}$

The set of modal properties, MODAL, are a philosophically more interesting case in point. Consider the following claim about the structure of the realm of possible worlds:

\section{Universal Access}

$\forall w \forall w^{\prime}\left(w\right.$ has access to $\left.w^{\prime}\right)$

If every world is accessible from every world, no two worlds disagree in their distribution of MODAL-properties, since every possible object will have the same MoDAL-properties at every possible world. Thus, no two worlds disagree in their distribution of MODAL-properties without disagreeing in their distribution of any other properties: MODAL-properties should trivially supervene on any set of properties. If Universal Access if false, on the other hand, and some worlds are not mutually accessible (perhaps because accessibility is not transitive), there will be worlds that differ in their distribution of Modal-properties. Distributional differences in MODAL-properties go together with the falsity of Universal Access.

\footnotetext{
${ }^{12}$ Kim's original notion of world-agreement and the corresponding notion of global supervenience does not share this difficulty. In this respect at least, the new understanding appears to have been a change for the worse.
} 
But according to Weak Global Supervenience this is not the case. MODALproperties do not trivially weak-globally supervene on any set of properties whether or not accessibility is universal. For, consider a world at which only a poached egg exists, $w$, and one at which only Socrates exists, $w^{\prime}$. The egg presumably does not have the property of being possibly human, while Socrates certainly does. Thus, there is no modal-property-preserving bijection between the domain of $w(=\{$ the egg $\})$ and the domain of $w^{\prime}$ $(=\{$ Socrates $\})$. Thus, MoDAL-properties do not weak-globally supervene (in the sense of Weak Global Supervenience) on any set of properties the egg and Socrates have in common.

Objection: The restriction in purview of Weak Global Supervenience to a world's existents is anything but arbitrary, but rather captures a reasonable interpretation of 'the distribution of $A$-properties at a world' in the informal global supervenience slogan, which refers to the distribution of $A$-properties among the individuals at a world. ${ }^{13}$ Reply: A phrase like 'the distribution of $A$-properties among the individuals at $w$ ' allows for at least two interpretations only one of which supports the objection. Very roughly, we may group the world-relativisation 'at $w$ ' with 'the distribution of $A$-properties' or with 'individuals'. The second option, on which we may be understood to talk merely about the individuals that exist at $w$, straightforwardly supports the objection. However, it is the first that conforms to the idea of supervenience of comparing worlds as to what goes on at them with respect to certain properties. Once it is granted that there are properties that are not existence-entailing, this will have to include considering whether these properties are exemplified at the relevant worlds by individuals that do not exist at them. Now, there may be interesting forms of restricted global supervenience, where only bijections between proper subclasses of individuals are taken into account: bijections between the concreta of the respective worlds, or between things that exist at them, for example. But such notions will merely be thematic restrictions of the underlying unrestricted notion to be introduced presently.

\section{World-Agreement Revised}

The problem with World-Agreement (as previously defined) is that it ignores the non-existents at a world. It does so by only considering world-domains, since a possible object $x$ is a member of $w$ 's world-domain if and only if $x$ exists at $w$. The fix to include a world's non-existents for comparison is

\footnotetext{
${ }^{13}$ Thanks to a reviewer for this journal for pushing me on this objection.
} 
obvious: instead of considering $A$-preserving bijections from world-domain to world-domain, we should consider $A$-preserving bijections from the universal domain - the union of all world-domains - to itself, that is, we should consider $A$-preserving permutations of $\mathfrak{D}$. This gives rise to the following proposal:

\section{World-Agreement!}

$w$ and $w^{\prime}$ agree! in their distribution of $A$-properties $\leftrightarrow_{\mathrm{df} .} \exists f$

(i) $\quad f: \mathfrak{D} \mapsto \mathfrak{D}$;

(ii) $f$ is bijective;

(iii) $\forall P \in A \forall x \in \mathfrak{D}$ (at $w, x$ has $P \leftrightarrow$ at $w^{\prime}, f(x)$ has $P$ ).

If a function satisfies (i) and (ii) it is a permutation of the universal domain. If it satisfies (iii) with respect to $w$ and $w^{\prime}$, it is $A$-preserving with respect to $w$ and $w^{\prime}$. Plugging this new understanding of what it means for worlds to agree in their distribution of some kind of properties into our informal understanding of global supervenience we get

\section{Weak Global Supervenience!}

$A$-properties weak-globally! supervene on $B$-properties $\leftrightarrow_{\mathrm{df}}$

For any worlds $w$ and $w^{\prime}$ : If there is a permutation of $\mathfrak{D}$ that is $B$-preserving with respect to $w$ and $w^{\prime}$, there is a permutation of $\mathfrak{D}$ that is $A$-preserving with respect to $w$ and $w^{\prime}$.

Clearly, World-Agreement! and, correspondingly, Weak Global Supervenience! avoid the pitfalls of their exclamation-mark-less variants. Worlds at only one of which Socrates exists do not agree! in their distribution of $P$ (the property of being such that one exists or Socrates does), since every permutation of $\mathfrak{D}$ will map some objects that do not have $P$ at the Socrates-less world to objects that have $P$ at the other, thus avoiding trivial weak-global! supervenience of $\{P\}$. And only if accessibility is less than universal do MODAL-properties fail to trivially weak-globally! supervene on any set of properties. For, if Universal Access holds, every possible object has the same MoDAL-properties at every possible word, and, thus, the identity permutation will map objects with the very same MODAL-properties to each other with respect to any two worlds. This is all as it should be. ${ }^{14}$

\footnotetext{
${ }^{14}$ Analogous modifications can be made to yield notions of intermediate!-global and strong!-global supervenience. Other than its exclamation-mark-less variant, immediate!global will sustain the intuition that MODAL-properties globally supervene even on a universally exemplified property (e.g. on the property of being self-identical), if Universal Access holds, while they do not so supervene if Universal Access fails. This is the less striking direct argument mentioned in fn. 6 above for replacing the received notion of intermediate global supervenience.
} 
Moreover, even a disbeliever in non-existence-entailing properties should prefer World-Agreement! and Weak Global Supervenience! (or, rather, slight modifications thereof) on account of their theoretical neutrality. For, if there are non-existence-entailing properties they, rather than their exclamationmark-less variants yield the correct results. And if there are only existenceentailing properties, World-Agreement and World-Agreement! (and, thus, Weak Global Supervenience and Weak Global Supervenience!) yield the same results. Therefore, it is theoretically prudent to bet on the notions of world-agreement and global supervenience introduced in this section rather than on their better-known variants. ${ }^{15}$

The equivalence claim can be easily seen to be correct for a slight modification of World-Agreement! that requires worlds that agree in their $A$ properties to have the same number of inhabitants. Call the result WorldAgreement!!. Since this requirement has no independent motivation, it was not included before. ${ }^{16}$ Once it is in place, though, we can see that two worlds agree just in case they agree!!, provided that all properties are existence-entailing.

$[\rightarrow]$ Suppose that $w$ and $w^{\prime}$ agree in their distribution of $A$-properties. Then there will be an $A$-preserving bijection from the domain of $w$ to the domain of $w^{\prime}$. Call it $f$. We can extend $f$ to a permutation of the universal domain, $f^{\prime}$, by letting $f^{\prime}$ map the non-existents of $w$ to the non-existents of $w^{\prime}$ in a one-one fashion. Since, by assumption, all $A$-properties are existence-entailing, none of the non-existents have any $A$-properties. Since $f^{\prime}$ extends $f$, all existents of $w$ get mapped to existents of $w^{\prime}$ with the same $A$-properties. Consequently, $f^{\prime}$ is $A$-preserving, and $w$ and $w^{\prime}$ agree!! in their distribution of $A$-properties.

$[\leftarrow]$ Suppose that $w$ and $w^{\prime}$ agree!! in their distribution of $A$-properties. Then (i) there is a permutation $f$ of the universal domain that is $A$-preserving and (ii) $w$ and $w^{\prime}$ have the same number of inhabitants. By (ii), there are various bijections between the world-domains of $w$ and $w^{\prime}$. Consider one that (a) agrees with $f$ whenever $f$ maps an inhabitant of $w$ to an inhabitant of $w^{\prime}$, and (b) maps every remaining inhabitant of $w$ to a remaining inhabitant of $w^{\prime}$ in a one-one fashion. Call it $f^{\prime}$. Since $w$ and $w^{\prime}$ have the same number of inhabitants, such a construction is possible. It is easy to see that $f^{\prime}$ is $A$-preserving. For, suppose $f^{\prime}$ maps $x$ to $y$. This is either an (a)- or a (b)-case. If it is an (a)-case, by construction of $f^{\prime}, f(x)=y$. By

\footnotetext{
${ }^{15}$ Thanks to a reviewer for this journal for suggesting to include this discussion.

${ }^{16}$ Arguably, the restriction in purview to same numbered worlds highlights part of what is odd about World-Agreement.
} 
(i), $x$ has the same $A$-properties at $w$ that $y$ has at $w^{\prime}$. If it is a (b)-case, again by construction of $f^{\prime}$, for some $z$ that does not exist at $w^{\prime}, f(x)=z$, and for some $z^{\prime}$ that does not exist at $w, f\left(z^{\prime}\right)=y$. By the assumption that $A$-properties are existence-entailing, $z$ does not have any $A$-properties at $w^{\prime}$ and $z^{\prime}$ does not have any at $w$. By (i), $x$ does not have any $A$-properties at $w$ and $y$ does not have any at $w^{\prime}$. Thus, $f^{\prime}$ is an $A$-preserving bijection from $\mathfrak{d}(w)$ to $\mathfrak{d}\left(w^{\prime}\right): w$ and $w^{\prime}$ agree in their distribution of $A$-properties.

Since World-Agreement and World-Agreement!! yield the same results, provided that there are no existence-entailing properties, so do the corresponding notions of global supervenience defined in their terms. Since nothing in what follows hinges on the restriction to same-numbered worlds, I will revert to speaking about the simpler notions explicitly defined above.

World-Agreement! and Weak Global Supervenience! are preferable to World-Agreement and Weak Global Supervenience respectively, since the former are able to deal with non-existence-entailing properties. In addition, the revised notion of supervenience allows us to counter an argument to the effect that global supervenience is theoretically dispensable. I end by presenting the argument and indicating where the argument fails for weakglobal! supervenience.

\section{The Entailment Claim}

Ever since Kim (1984: 168) presented a flawed attempted proof to the effect that global supervenience (as defined by him) entails strong individual supervenience, this question has been controversially discussed in the literature. Call the claim that (some form of) global supervenience entails strong supervenience the Entailment Claim (with respect to that form of global supervenience). The received opinion is that the Entailment Claim is false with respect to all forms of global supervenience, but that a certain restriction thereof is true even with respect to weak global supervenience: when $A$ and $B$ are restricted to intrinsic properties, $A$-properties strongly supervene on $B$-properties if the former weak-globally supervene on the latter. Call this claim the Restricted Entailment Claim.

Kim (1993a: 170) conjectured that at least the Restricted Entailment Claim is true. Recently, Karen Bennett (2004: Appendix) has provided a proof. ${ }^{17}$ She uses this result as part of an argument to the effect that,

\footnotetext{
${ }^{17}$ Bennett (2004) attempts to prove a slightly stronger claim-unsuccessfully, as Moyer (2008: fn. 26) has pointed out. However, an obvious variant of Bennett's proof establishes that the weaker claim is true, as will be shown presently.
} 
'[d]espite its apparent usefulness, then, global supervenience [is] little more than a chimera' (Bennett 2004: 501). The friend of global supervenience may be happy to learn that Bennett's argument does not apply to the notions of global supervenience defined above.

In this section I sketch the argument for Restricted Entailment with respect to weak global supervenience. I then point out at which point the corresponding argument with respect to weak global! supervenience breaks down. I end by showing that modal properties provide a counterexample both to the Entailment Claim and its restriction. Not only is the Restricted Entailment Claim unproven, it is false.

Let us start by getting clear about the notion of strong individual supervenience at issue. $A$-properties individually supervene on $B$-properties just in case an individual's $B$-properties fix its $A$-properties. The definition of strong individual supervenience spells out this intuitive idea as follows:

\section{Strong Supervenience}

$A$-properties strongly supervene on $B$-properties $\leftrightarrow_{\mathrm{df}}$. $\forall w \forall w^{\prime} \forall x \forall y\left(\forall P\left(P\right.\right.$ is a $B$-property $\rightarrow\left(\right.$ at $w, x$ has $P \leftrightarrow$ at $w^{\prime}, y$ has $\left.\left.P\right)\right) \rightarrow$ $\forall Q\left(Q\right.$ is an $A$-property $\rightarrow\left(\right.$ at $w, x$ has $Q \leftrightarrow$ at $w^{\prime}, y$ has $\left.\left.\left.Q\right)\right)\right)$

In words: $A$-properties strongly supervene on $B$-properties just in case whenever possible individuals are $B$-indiscernible at some worlds, they are $A$-indiscernible at these worlds as well. ${ }^{18}$

At the beginning of section 1 above we have already seen a counterexample against the unrestricted Entailment Claim with respect to weak global supervenience: the MostMAss-property weak-globally but not strongly supervenes on MASs-properties. However, this counterexample is similar to the one provided by Paull and Sider (1992: §3) in making essential use of extrinsic supervenience candidates. Consequently, it does not speak against the Restricted Entailment Claim.

Indeed, there is a relatively simple argument for the Restricted Entailment Claim with respect to weak global supervenience. It assumes the following quite plausible recombination principle (cp. Paull and Sider 1992: 838f.):

\section{Isolation}

For any world $w$ and individual $x$, if $x$ exists at $w$, then there is a world $w^{\prime}$ and an individual $y$ such that

(i) only $y$ ( $y$ 's parts and objects whose existence is necessitated by the existence of $y$ or $y$ 's parts) exists at $w^{\prime}$; and

\footnotetext{
${ }^{18}$ Cf., e.g., Kim (1987: 317).
} 
(ii) $\quad y$ at $w^{\prime}$ is a duplicate of $x$ at $w$.

Intuitively, Isolation says that whenever we have an individual existing at a world, there might have been an isolated duplicate of that thing. ${ }^{19}$ Now, according to Paull and Sider, 'duplicates are exactly qualitatively similar considered "as they are in themselves" and not in relation to other things'. Think, for instance, of the Mona Lisa and a perfect facsimile, Fona. Mona and Fona share a great many properties: they have the same size and mass as well as the same colour distribution, for instance. But they may also differ in certain respects: clearly, only one of them is Mona, Fona is worth considerably less than Fona and only Mona was painted by Leonardo da Vinci. Let us further accept the claim that duplicates can never differ in intrinsic properties as a (partial) elucidation of what it is for a property to be intrinsic. $^{20}$

Given Isolation it is easy to show that whenever $A$ - and $B$-properties are intrinsic, $A$-properties strongly supervene on $B$-properties provided that they weak-globally supervene on $B$-properties. Suppose that (i) $A$ and $B$ only contain intrinsic properties and that (ii) $A$-properties do not strongly supervene on $B$-properties. Then, by (ii), (iii) there are individuals $a$ and $b$ and worlds $w_{1}$ and $w_{2}$ such that $a$ at $w_{1}$ and $b$ at $w_{2}$ are $A$-discernible but $B$-indiscernible. By Isolation, (iv) there are further worlds $w_{3}$ and $w_{4}$ at which only isolated duplicates of $a$ and $b$ exist. Call them $c$ and $d$ respectively. Thus, by (i), $c$ at $w_{3}$ is $A$ - and $B$-indiscernible from $a$ at $w_{1}$ as is $d$ at $w_{4}$ from $b$ at $w_{2}$. Consequently, by (iii), (v) $c$ at $w_{3}$ is $A$-discernible but $B$-indiscernible from $d$ at $w_{4}$. By (iv), (vi) there is only one bijection between the domains of $w_{3}$ and $w_{4}$ : the function $f$ that maps $c$ to $d$. By $(\mathrm{v})$, $f$ is $B$-preserving but not $A$-preserving. By (vi), there is no other bijection between the two domains that is $A$-preserving either. Thus, $A$-properties do not weak-globally supervene on $B$-properties. Contraposition yields the desired result: the Restricted Entailment Claim is true with respect to weak global supervenience.

It is easy to see, however, that the analogous proof attempt for weakglobal! supervenience does not go through. For, the Bennett-style proof relies on there being just one bijection between the world-domains of $w_{3}$ and $w_{4}$, the one that maps $c$ to $d$. Only this allowed us to conclude that there

\footnotetext{
${ }^{19}$ Or rather: a duplicate that is isolated as much as possible. This is meant to be covered by the parenthesis in (i). In order to avoid unnecessary clutter I will ignore difficulties concerning composite objects and ontological dependence in the main text.

${ }^{20}$ Cp. Paull and Sider (1992: 838) For more on the intrinsic/extrinsic divide and its relation to duplication see, e.g., Weatherson (2008).
} 
is no relevant $A$-preserving bijection. But since the purview of weak-global! supervenience is not restricted to the existents of worlds, far more bijections are relevant to weak global! supervenience of $A$ - on $B$-properties. And the argument does nothing to show that none of them will be $A$-preserving.

Indeed, no sound argument will show this, since it is not generally true. We have already come across the makings of a counterexample against the Restricted Entailment Claim with respect to weak-global! supervenience. For, recall that, on the assumption of Universal Access, MODAL-properties trivially weak-globally! supervene on any set of properties, for instance on the set of mass properties MASS. But MODAL-properties do not strongly supervene on all sets of intrinsic properties. In particular, MODAL-properties do not strongly supervene on MASs-properties. My desk and I, let's assume, have the same mass at the actual world. However, I am possibly human while the desk is not. Thus, I am MAss-indiscernible but MoDAL-discernible from my desk at the actual world. MODAL- and MASs-properties provide a counterexample to the Entailment Claim, given Universal Access.

The case provides also the basis for a counterexample to the Restricted Entailment Claim. For, modal properties are not extrinsic qua modal properties. To be sure, Modal includes some extrinsic properties, e.g. the property of possibly being married. But such modal properties plausibly inherit their extrinsicness from the properties they are modalizations of, and, more importantly, intrinsic properties plausibly have intrinsic modalizations. ${ }^{21}$

For instance, there seems to be no reason to think that the property of possibly having a mass of $2 \mathrm{~kg}$ is extrinsic (assuming that the property of having a mass of $2 \mathrm{~kg}$ isn't).

Firstly, it is in no obvious way a relational property, as opposed to such paradigm examples of extrinsic properties as the property of being married. The only reason I can see for thinking otherwise is the popular doctrine that the best semantics for modal sentences explains their truth in terms of quantification over possible worlds. But this alone is not a good reason to think that modal properties are relational, otherwise we would have an analogous reason for thinking that all properties are. For, it is also commonly held that the best semantics for simple subject-predicate sentences explains their truth in terms of the semantic values of predicates, e.g., sets or properties. That is, a sentence of the form ' $a$ is $F$ ' is often supposed to be true (if it is) because $a$ is an element of the set of all $F$ s, or because $a$ has the property of being $F$. But ' $a$ is an element of the set of

\footnotetext{
${ }^{21}$ This claim is stronger than it needs to be. For the argument to go through, we only need one intrinsic modal property that is not shared by everything.
} 
all $F$ s' and ' $a$ has the property of being $F$ ' ascribe relational properties, to wit: being an element of $\{x: x$ is $F\}$ and having the property of being $F$ respectively.

Secondly, duplicates cannot differ in the property of possibly having a mass of $2 \mathrm{~kg}$ : if a thing has it, all of its duplicates do, and if a thing lacks it, all of its duplicates lack it. Thus, the property of possibly having a mass of $2 \mathrm{~kg}$ has as good a claim to being an intrinsic property as the property of having a mass of $2 \mathrm{~kg}$.

We may then take the subset of MoDAL that includes only intrinsic properties, MODAL . MODALI includes the property of possibly having a mass of $2 \mathrm{~kg}$ among many others. Since MODALI is a subset of ModAL and MODAL-properties trivially weak-globally! supervene on any set of properties, MODALI-properties trivially weak-globally! supervene on any set of properties, e.g., on MASS-properties. But MODALI-properties do not strongly supervene on MAss-properties. Thus MODAL I and MAss provide a counterexample even to the Restricted Entailment Claim, given that every world is accessible from every other.

Now, this is a conditional result on the assumption of Universal Access. However, this assumption is quite hard to deny without denying the correctness of S5. For, although there are S5 models that violate it, Universal Access still underlies S5 in the sense that for every S5 model $M$ there is one in which Universal Access holds and at whose designated world a formula is true just in case it is true in $M$. If $\mathrm{S} 5$ is correct, each world is either accessible to the actual world or not even mediately accessible from it (i.e. via a chain of accessible worlds). If there were any worlds that are not even mediately accessible, they would be the ones to falsify Universal Access. But they would also be absolutely irrelevant to the truth of any modal statements framed in terms of possibility and necessity. It is very hard to believe in such inaccessible worlds, if S5 correct. If S5 is not correct, on the other hand, there will be inaccessible worlds that are mediately accessible. Consequently, there will be modal properties that things have only contingently. However, it is anything but clear that any of the arguments against S5 support the view that all modal properties of the kind required for the counterexample are contingent. $^{22}$ If not, we can simply restrict MoDAL further to exclude contingently exemplifiable modal properties. There is, in conclusion, little relief the friend of Restricted Entailment can hope for from

\footnotetext{
${ }^{22}$ E.g. the argument developed in Chandler (1976) and Salmon (1982: §28) justifies the thesis of contingent exemplification only for a very special class of properties: properties of the kind of possibly being initially made of a certain material.
} 
the denial of Universal Access.

Let me sum up. In this paper I have argued that the predominant notions of global supervenience are inadequate, since they rely on a notion of world-agreement whose purview is too restrictive. The notion of worldagreement employed should be able to deal with properties that are not existence-entailing. World-Agreement! fits the bill. The resulting notions of global supervenience have another point in their favour: they avoid an argument to the effect that they are theoretically dispensable, since equivalent with strong supervenience. ${ }^{23}$ Pace Bennett, global supervenience may have important philosophical work to do. Global! supervenience notions are better equipped to do this work than their more familiar relatives. ${ }^{24}$

\section{References}

Bennett, K. 2004: 'Global Supervenience and Dependence'. Philosophy and Phenomenological Research 68, pp. 501-529.

Chandler, H. S. 1976: 'Plantinga and the Contingently Possible'. Analysis 36, pp. 106-109.

Correia, F. 2005: Existential Dependence and Cognate Notions. Munich: Philosophia Verlag.

Fine, K. 1985: 'Plantinga on the Reduction of Possibilist Discourse'. In Tomberlin, J. E. and P. van Inwagen (eds.): Alvin Plantinga, Dordrecht: D. Reidel, pp. 145-186, reprinted in Fine (2005: 176-213). Page references are to the reprint.

2005: Modality and Tense. Philosophical Papers. Oxford: Clarendon Press.

\footnotetext{
${ }^{23}$ I have not shown, of course, that the new supervenience notions avoid all arguments targeted at the more familiar notions. Notable further difficulties that would need to be addressed in a full treatment are (a) worries concerning structural features raised, e.g., in Leuenberger (2009: §2-3) and (b) the worry that, like all other forms of supervenience defined in terms of modal notions, global! supervenience does not constitute a strong form of dependence (cp. e.g. Kim 1990; Correia 2005: ch. 6). I have to leave discussion to future research.

${ }^{24}$ Thanks to the participants of the Hamburg Colloquium on Metaphysics and the Philosophy of Language in the summer term 2012, to audiences in London, Stockholm, Barcelona and Konstanz as well as to Mark Kalderon, Lee Walters and my co-Phloxers Nick Haverkamp, Miguel Hoeltje, Stephan Krämer, Benjamin Schnieder and Moritz Schulz for discussion of the material at various stages of completion. Thanks are also due to two anonymous referees for this journal for helpful comments.
} 
Fitting, M. and R. L. Mendelsohn 1998: First-Order Modal Logic, Synthese Library. Studies in Epistemology, Logic, Methodology, and Philosophy of Science, vol. 277. Dordrecht: Kluwer Academic Publishers.

Kim, J. 1984: 'Concepts of Supervenience'. Philosophy and Phenomenological Research 45, pp. 153-176.

1987: "Strong" and "Global" Supervenience Revisited'. Philosophy and Phenomenological Research 48, pp. 315-326.

1988: 'Supervenience for Multiple Domains'. Philosophical Topics 16, pp. 129-150.

1990: 'Supervenience as a Philosophical Concept'. Metaphilosophy 21, pp. $1-27$.

— 1993a: 'Postscripts on Supervenience'. In Kim (1993b), pp. 161-171.

1993b: Supervenience and Mind. Selected Philosophical Essays. Cambridge: Cambridge University Press.

Leuenberger, S. 2009: 'What is Global Supervenience?' Synthese 170, pp. $115-129$.

Lewis, D. 1986: On the Plurality of Worlds. Oxford: Blackwell.

Linsky, B. and E. N. Zalta 1994: 'In Defense of the Simplest Quantified Modal Logic'. Philosophical Perspectives 8, pp. 431-458.

McLaughlin, B. P. 1995: 'Varieties of Supervenience'. In Savellos, E. E. and Ü. D. Yalçin (eds.): Supervenience: New Essays, Cambridge: Cambridge University Press, pp. 16-59.

1997: 'Supervenience, Vagueness, and Determination'. Nô̂s 31, pp. 209-230.

Moyer, M. 2008: 'Weak and Global Supervenience are Strong'. Philosophical Studies 138, pp. 125-150.

Paull, R. C. and T. Sider 1992: 'In Defense of Global Supervenience'. Philosophy and Phenomenological Research 52, pp. 833-854.

Plantinga, A. 1983: 'On Existentialism'. Philosophical Studies 44, pp. 1-20.

Salmon, N. 1982: Reference and Essence. Oxford: Basil Blackwell. 
Shagrir, O. 2002: 'Global Supervenience, Coincident Entities and AntiIndividualism'. Philosophical Studies 109, pp. 171-196.

Sider, T. 1999: 'Global Supervenience and Identity across Times and Worlds'. Philosophy and Phenomenological Research 59, pp. 913-937.

Stalnaker, R. C. 1996: 'Varieties of Supervenience'. Philosophical Perspectives 10, pp. 221-241.

Weatherson, B. 2008: 'Intrinsic vs. Extrinsic Properties'. In Zalta, E. N. (ed.): The Stanford Encyclopedia of Philosophy, fall 2008 ed. Available at http://plato.stanford.edu/archives/fall2008/ entries/intrinsic-extrinsic/

Williamson, T. 2002: 'Necessary Existents'. In O'Hear, A. (ed.): Logic, Thought and Language, Cambridge: Cambridge University Press, pp. 233251. 